\title{
PERIODICITY OF SOLUTIONS FOR A SYSTEM OF NONLINEAR INTEGRO-DIFFERENTIAL EQUATIONS
}

\author{
MOUATAZ BILLAH MESMOULI, ABDELOUAHEB ARDJOUNI AND AHCENE \\ DJOUDI
}

ABSTRACT. In this paper, we study the existence of periodic solutions of the nonlinear system of integro-differential equations

$$
\frac{d}{d t} x(t)=-\int_{t-\tau(t)}^{t} A(t, s) x(s) d s+Q(t, x(t), x(t-\tau(t))), t \in \mathbb{R} .
$$

In the process we use the fundamental matrix solution to convert the given integro-differential equation into an equivalent integral equation. Then by using Krasnoselskii's fixed point theorem we obtain the existence of periodic solution and by contraction mapping principle we obtain the uniqueness. An application in two dimension is given.

\section{INTRODUCTION}

A qualitative analysis of periodicity, positivity and stability of solutions of neutral differential equations has been with delay studied extensively by many authors. We refer the readers to $[1]-[3],[6]-[14],[16,19]$ and references therein for a wealth of reference materials on the subject.

Recently, E. Yankson in [19] studied the existence and uniqueness of a periodic solution of the system of differential equations

$$
\frac{d}{d t} x(t)=A(t) x(t-\tau), t \in \mathbb{R},
$$

where $A(\cdot)$ is an $n \times n$ matrix with continuous real-valued functions as its elements and $\tau$ is a positive constant.

2010 Mathematics Subject Classification. 34A34, 35K13, 45J05, 47H10.

Key words and phrases. Krasnoselskii's theorem, contraction, neutral differential equation, integral equation, periodic solution, fundamental matrix solution, Floquet theory.

Copyright (c) 2015 by ANUBiH. 
In the present paper, we study the existence of periodic solutions of the system of nonlinear integro-differential equations

$$
\frac{d}{d t} x(t)=-\int_{t-\tau(t)}^{t} A(t, s) x(s) d s+Q(t, x(t), x(t-\tau(t))), t \in \mathbb{R},
$$

where $A(\cdot, \cdot)$ is a nonsingular $n \times n$ matrix with continuous real-valued functions as its elements and $G: \mathbb{R} \times \mathbb{R}^{n} \times \mathbb{R}^{n} \rightarrow \mathbb{R}^{n}$ is a continuous function. In the analysis we use the fundamental matrix solution of $x^{\prime}(t)=D(t) x(t)$ coupled with Floquet theory, where $D(\cdot)$ is a nonsingular $n \times n$ matrix with continuous real-valued functions as its elements used to invert the system (1.2) into an integral system. Then, we employ the Krasnoselskii's fixed point theorem to show the existence of periodic solutions of system (1.2). The obtained integral system is the sum of two mappings, one is a compact operator and the other is a contraction. Also, transforming system (1.2) to an integral system enables us to show the uniqueness of the periodic solution by appealing to the contraction mapping principle.

The organization of this paper is as follows. In Section 2, we present some remarks from [19], the inversion of (1.2) and the fixed point theorems that are used to prove the existence and uniqueness of solutions to equation (1.2). In Section 3, we present our main results. A two dimensional application is the topic of Section 4.

\section{Preliminaries}

For the definitions of different notions used throughout this paper we refer to $[2,4,15,17,18]$. For $T>0$ define $\mathcal{C}_{T}=\left\{\phi: \phi \in C\left(\mathbb{R}^{n}\right), \phi(t+T)=\phi(t)\right.$, $t \in \mathbb{R}\}$ where $C\left(\mathbb{R}^{n}\right)$ is the space of all $n$-vector continuous functions. Then $\mathcal{C}_{T}$ is a Banach space when it is endowed with the supremum norm

$$
\|x(\cdot)\|=\max _{t \in[0, T]}|x(t)|,
$$

where $|\cdot|$ denotes the infinity norm for $x \in \mathbb{R}^{n}$. Also, if $A$ is an $n \times n$ real matrix, then we define the norm of $A$ by

$$
\|A\|=\max _{t \in[0, T]}\left(\max _{1 \leq i \leq n} \sum_{j=1}^{n}\left|a_{i j}(t)\right|\right) .
$$

Definition 2.1. If the matrix $D(\cdot)$ is periodic of period $T$, then the linear system

$$
y^{\prime}(t)=D(t) y(t)
$$

is said to be noncritical with respect to $T$ if it has no periodic solutions of period $T$ except for the trivial solution $y=0$. 
In this paper we assume that

$A(t+T, s+T)=A(t, s), \tau(t+T)=\tau(t) \geq \tau^{*}>0$ for all $t, s \in \mathbb{R}$,

where $\tau$ is twice continuously differentiable with $\tau^{*}$ is constant and

$$
\tau^{\prime}(t) \neq 1 \text {, for all } t \in \mathbb{R} \text {. }
$$

For $t \in \mathbb{R}, x, y, z, w \in \mathbb{R}^{n}$, the function $Q(t, x, y)$ is periodic in $t$ of period $T$, it is also globally Lipschitz continuous in $x$ and $y$. That is

$$
Q(t+T, x, y)=Q(t, x, y), t \in \mathbb{R}
$$

and there are positive constants $k_{1}$ and $k_{2}$ such that

$$
|Q(t, x, y)-Q(t, z, w)| \leq k_{1}\|x-z\|+k_{2}\|y-w\|, t \in \mathbb{R}, x, y, z, w \in \mathbb{R}^{n} .
$$

Throughout this paper we assume that the system (2.1) is noncritical. Next we state some known results [4] about system (2.1). Let $K(t)$ represents the fundamental matrix solution of $(2.1)$ with $K(0)=I$, where $I$ is the $n \times n$ identity matrix. Then:

a. $\operatorname{det} K(t) \neq 0, t \in \mathbb{R}$,

b. There exists a constant matrix $B$ such that $K(t+T)=K(t) e^{T B}$, $t \in \mathbb{R}$, by Floquet theory.

c. System (2.1) is noncritical if and only if $\operatorname{det}(I-K(T)) \neq 0$.

The following lemmas are fundamental in our results.

Lemma 2.1. The equation (1.2) is equivalent to

$$
\begin{aligned}
& \frac{d}{d t} x(t)=\frac{d}{d t} \int_{t-\tau(t)}^{t} B(t, s) x(s) d s+B(t, t-\tau(t))\left(1-\tau^{\prime}(t)\right) x(t-\tau(t)) \\
& +Q(t, x(t), x(t-\tau(t))),
\end{aligned}
$$

where

$$
B(t, s)=\int_{t}^{s} A(u, s) d u \text { and } B(t, t-\tau(t))=\int_{t}^{t-\tau(t)} A(u, t-\tau(t)) d u
$$

Proof. Differentiating the first integral term in (2.6), we obtain

$$
\begin{aligned}
& \frac{d}{d t} \int_{t-\tau(t)}^{t} B(t, s) x(s) d s \\
& =B(t, t) x(t)-B(t, t-\tau(t))\left(1-\tau^{\prime}(t)\right) x(t-\tau(t))+\int_{t-\tau(t)}^{t} \frac{\partial}{\partial t} B(t, s) x(s) d s .
\end{aligned}
$$

Substituting this into (2.6), it follows that (2.6) is equivalent to (1.2) provided $B$ satisfies the following conditions

$$
B(t, t)=0 \quad \text { and } \quad \frac{\partial}{\partial t} B(t, s)=-A(t, s) .
$$


This equality implies

$$
B(t, s)=-\int_{0}^{t} A(u, s) d u+\phi(s)
$$

for some function $\phi$, and $B(t, s)$ must satisfy

$$
B(t, t)=-\int_{0}^{t} A(u, t) d u+\phi(t)=0 .
$$

Therefore,

$$
\phi(t)=\int_{0}^{t} A(u, t) d u
$$

Substituting this into (2.8), we obtain

$$
B(t, s)=-\int_{0}^{t} A(u, s) d u+\int_{0}^{s} A(u, s) d u=\int_{t}^{s} A(u, s) d u .
$$

This definition of $B$ satisfies (2.7). Consequently, (1.2) is equivalent to (2.6).

Remark 2.1. According to the notation used by E. Yankson in [19], in equation (1.1) it is assumed that there is a nonsingular $n \times n$ matrix $G(\cdot)$ with continuous real-valued functions as its elements such that

$$
\frac{d}{d t} x(t)=G(t) x(t)-\frac{d}{d t} \int_{t-\tau}^{t} G(u) x(u) d u+[A(t)-G(t-\tau)] x(t-\tau) .
$$

But this conditions is not necessary and we can replace $G(\cdot)$ by $A(\cdot)$ because $A(t-\tau)$ exists. However, in the present work, this condition is removed and we assume that $A(\cdot, \cdot)$ is a nonsingular $n \times n$ matrix. So, we can rewrite equation (1.2) as

$$
\frac{d}{d t} x(t)=D(t) x(t-\tau(t))+\frac{d}{d t} \int_{t-\tau(t)}^{t} B(t, s) x(s) d s+Q(t, x(t), x(t-\tau(t)))
$$

with $D(t)=B(t, t-\tau(t))\left(1-\tau^{\prime}(t)\right)$ and we replace $G(\cdot)$ in [19] by $D(\cdot)$.

Lemma 2.2. Suppose (2.2), (2.3) and (2.4) hold. If $x \in \mathcal{C}_{T}$, then $x$ is a solution of equation (1.2) if and only if

$$
\begin{aligned}
& x(t)=\int_{t-\tau(t)}^{t}(B(t, s)-D(s)) x(s) d s \\
& +K(t) U(T) \int_{t}^{t+T} K^{-1}(s) D(s) \int_{s-\tau(s)}^{s}(B(s, u)-D(u)) x(u) d u d s \\
& +K(t) U(T) \int_{t}^{t+T} K^{-1}(s)(F(s) x(s-\tau(s))+Q(s, x(s), x(s-\tau(s)))) d s,
\end{aligned}
$$


where

and

$$
U(T)=\left(K^{-1}(T)-I\right)^{-1}
$$

$$
F(t)=D(t)-\left(1-\tau^{\prime}(t)\right) D(t-\tau(t)) .
$$

Proof. First, since $A(\cdot, \cdot)$ is a nonsingular $n \times n$ matrix and condition (2.3), one can easily see that $D(\cdot)$ is also nonsingular. Then, let $x \in \mathcal{C}_{T}$ be a solution of (1.2) and $K(t)$ a fundamental system of solutions for (2.1). Rewrite the equation (1.2) as

$$
\begin{aligned}
& \frac{d}{d t} x(t)=D(t) x(t)-D(t) x(t)+\left(1-\tau^{\prime}(t)\right) D(t-\tau(t)) x(t-\tau(t)) \\
& +\left[D(t)-\left(1-\tau^{\prime}(t)\right) D(t-\tau(t))\right] x(t-\tau(t))+\frac{d}{d t} \int_{t-\tau(t)}^{t} B(t, s) x(s) d s \\
& +Q(t, x(t), x(t-\tau(t))) \\
& =D(t) x(t)-\frac{d}{d t} \int_{t-\tau(t)}^{t} D(u) x(u) d u+\frac{d}{d t} \int_{t-\tau(t)}^{t} B(t, s) x(s) d s \\
& +\left[D(t)-\left(1-\tau^{\prime}(t)\right) D(t-\tau(t))\right] x(t-\tau(t))+Q(t, x(t), x(t-\tau(t))) .
\end{aligned}
$$

For the sake of simplicity, we let

$$
D(t)-\left(1-\tau^{\prime}(t)\right) D(t-\tau(t))=F(t)
$$

and

$$
\begin{aligned}
\Xi(t)=D(t) \int_{t-\tau(t)}^{t}(B(t, u) & -D(u)) x(u) d u \\
& +F(t) x(t-\tau(t))+Q(t, x(t), x(t-\tau(t))) .
\end{aligned}
$$

Then

$$
\begin{aligned}
\frac{d}{d t}\left[x(t)-\int_{t-\tau(t)}^{t}\right. & (B(t, s)-D(s)) x(s) d s] \\
= & D(t)\left[x(t)-\int_{t-\tau(t)}^{t}(B(t, s)-D(s)) x(s) d s\right]+\Xi(t) .
\end{aligned}
$$

Since $K(t) K^{-1}(t)=I$, it follows that

$$
\begin{aligned}
0 & =\frac{d}{d t}\left[K(t) K^{-1}(t)\right]=D(t) K(t) K^{-1}(t)+K(t) \frac{d}{d t} K^{-1}(t) \\
& =D(t)+K(t) \frac{d}{d t} K^{-1}(t) .
\end{aligned}
$$

This implies

$$
\frac{d}{d t} K^{-1}(t)=-K^{-1}(t) D(t)
$$


If $x(\cdot)$ is a solution of $(1.2)$ with $x(0)=x_{0}$, then

$$
\begin{aligned}
& \frac{d}{d t}\left[K^{-1}(t)\left(x(t)-\int_{t-\tau(t)}^{t}(B(t, s)-D(s)) x(s) d s\right)\right] \\
& =\frac{d}{d t} K^{-1}(t)\left[x(t)-\int_{t-\tau(t)}^{t}(B(t, s)-D(s)) x(s) d s\right] \\
& +K^{-1}(t) \frac{d}{d t}\left[x(t)-\int_{t-\tau(t)}^{t}(B(t, s)-D(s)) x(s) d s\right] \\
& =-K^{-1}(t) D(t)\left[x(t)-\int_{t-\tau(t)}^{t}(B(t, s)-D(s)) x(s) d s\right] \\
& +K^{-1}(t)\left[D(t)\left(x(t)-\int_{t-\tau(t)}^{t}(B(t, s)-D(s)) x(s) d s\right)+\Xi(t)\right] .
\end{aligned}
$$

An integration of the above equation from 0 to $t$ yields

$$
\begin{aligned}
& x(t)=\int_{t-\tau(t)}^{t}(B(t, s)-D(s)) x(s) d s \\
+ & K(t)\left(x(0)-\int_{-\tau(0)}^{0}(B(0, s)-D(s)) x(s) d s\right)+K(t) \int_{0}^{t} K^{-1}(s) \Xi(s) d s .
\end{aligned}
$$

Since $x(T)=x_{0}=x(0)$, using $(2.10)$ we get

$$
\begin{aligned}
x(0)-\int_{-\tau(0)}^{0}(B(0, s) & -D(s)) x(s) d s \\
= & (I-K(T))^{-1} \int_{0}^{T} K(T) K^{-1}(s) \Xi(s) d u .
\end{aligned}
$$

A substitution of (2.11) into (2.10) yields

$$
\begin{aligned}
& x(t)=\int_{t-\tau(t)}^{t}(B(t, s)-D(s)) x(s) d s \\
& +K(t)(I-K(T))^{-1} \int_{0}^{T} K(T) K^{-1}(s) \Xi(s) d s+K(t) \int_{0}^{t} K^{-1}(s) \Xi(s) d s .
\end{aligned}
$$

We will now show that (2.12) is equivalent to (2.9). Since

$$
(I-K(T))^{-1}=\left(K(T)\left(K(T)^{-1}-I\right)\right)^{-1}=\left(K(T)^{-1}-I\right)^{-1} K(T)^{-1},
$$


the equation (2.12) becomes

$$
\begin{aligned}
& x(t)=\int_{t-\tau(t)}^{t}(B(t, s)-D(s)) x(s) d s \\
& +K(t)\left(K(T)^{-1}-I\right)^{-1} \int_{0}^{T} K^{-1}(s) \Xi(s) d s+K(t) \int_{0}^{t} K^{-1}(s) \Xi(s) d s,
\end{aligned}
$$

then

$$
\begin{array}{r}
x(t)=\int_{t-\tau(t)}^{t}(B(t, s)-D(s)) x(s) d s+K(t)\left(K(T)^{-1}-I\right)^{-1} \\
\cdot\left\{\int_{0}^{T} K^{-1}(s) \Xi(s) d s+\int_{0}^{t}\left(K(T)^{-1}-I\right) K^{-1}(s) \Xi(s) d s\right\} \\
=\int_{t-\tau(t)}^{t}(B(t, s)-D(s)) x(s) d s+K(t)\left(K(T)^{-1}-I\right)^{-1} \\
\cdot\left\{\int_{0}^{T} K^{-1}(s) \Xi(s) d s-\int_{0}^{t} K^{-1}(s) \Xi(s) d s+\int_{0}^{t} K(T)^{-1} K^{-1}(s) \Xi(s) d s\right\} \\
=\int_{t-\tau(t)}^{t}(B(t, s)-D(s)) x(s) d s+K(t)\left(K(T)^{-1}-I\right)^{-1} \\
\cdot\left\{\int_{t}^{T} K^{-1}(s) \Xi(s) d s+\int_{0}^{t} K(T)^{-1} K^{-1}(s) \Xi(s) d s\right\} .
\end{array}
$$

By letting $s=v-T$ and $U(T)=\left(K(T)^{-1}-I\right)^{-1}$, the above expression implies

$$
\begin{aligned}
x(t) & =\int_{t-\tau(t)}^{t}(B(t, s)-D(s)) x(s) d s+K(t) U(T) \int_{t}^{T} K^{-1}(s) \Xi(s) d s \\
& +K(t) U(T) \int_{T}^{t+T} K(T)^{-1} K^{-1}(v-T) \Xi(v-T) d v .
\end{aligned}
$$

By (b) we have $K(t-T)=K(t) e^{-T B}$ and $K(T)=e^{T B}$. Hence,

$$
K^{-1}(T) K^{-1}(v-T)=K^{-1}(v) .
$$

Consequently, since (2.2) and (2.4) hold, (2.13) becomes

$$
\begin{aligned}
x(t) & =\int_{t-\tau(t)}^{t}(B(t, s)-D(s)) x(s) d s \\
& +K(t) U(T) \int_{t}^{T} K^{-1}(s) \Xi(s) d s+K(t) U(T) \int_{T}^{t+T} K^{-1}(s) \Xi(s) d s .
\end{aligned}
$$


By combining the two integrals of equation (2.14), we can obtain easily the equation (2.9) The converse implication is straightforward. The proof is complete.

We end this section by stating the fixed point theorems that we employ to help us show the existence and uniqueness of solutions to equation (1.2) (see $[2,17])$.

Theorem 2.1 (Contraction mapping principle). Let $(\mathcal{X}, \rho)$ a complete metric space and let $P: \mathcal{X} \rightarrow \mathcal{X}$. If there is a constant $\alpha<1$ such that for $x, y \in \mathcal{X}$ we have

$$
\rho(P x, P y) \leq \alpha \rho(x, y),
$$

then there is one and only one point $z \in \mathcal{X}$ with $P z=z$.

Krasnoselskii (see [17]) combined the contraction mapping theorem and Schauder's theorem and formulated the following hybrid result.

Theorem 2.2 (Krasnoselskii). Let $\mathbb{M}$ be a closed bounded convex nonempty subset of a Banach space $(\mathcal{X},\|\cdot\|)$. Suppose that $R$ and $S$ map $\mathbb{M}$ into $\mathcal{X}$ such that

(i) $R$ is compact and continuous,

(ii) $S$ is a contraction mapping,

(iii) $x, y \in \mathbb{M}$, implies $R x+S y \in \mathbb{M}$,

Then there exists $z \in \mathbb{M}$ with $z=R z+S z$.

\section{Existence AND Uniqueness of PERIOdiC SOlUtions}

By applying Theorems 2.1 and 2.2, we obtain in this Section the existence and the uniqueness of periodic solutions for (1.2). So, let a Banach space $\left(\mathcal{C}_{T},\|\cdot\|\right)$ have a closed bounded convex subset of $\mathcal{C}_{T}$,

$$
\mathcal{M}=\left\{\varphi \in \mathcal{C}_{T},\|\varphi\| \leq L\right\}
$$

with $L>0$, and by the Lemma 2.2, let a mapping $\mathcal{H}$ be given by

$$
\begin{aligned}
& (\mathcal{H} \varphi)(t)=\int_{t-\tau(t)}^{t}(B(t, s)-D(s)) \varphi(s) d s \\
& +K(t) U(T) \int_{t}^{t+T} K^{-1}(s) D(s) \int_{s-\tau(s)}^{s}(B(s, u)-D(u)) \varphi(u) d u d s \\
& +K(t) U(T) \int_{t}^{t+T} K^{-1}(s)[F(s) \varphi(s-\tau(s))+Q(s, \varphi(s), \varphi(s-\tau(s)))] d s .
\end{aligned}
$$

We express equation (3.2) as

$$
\mathcal{H} \varphi=\mathcal{R} \varphi+\mathcal{S} \varphi
$$


where $\mathcal{R}, \mathcal{S}$ are given by

$$
\begin{aligned}
& (\mathcal{R} \varphi)(t) \\
& =K(t) U(T) \int_{t}^{t+T} K^{-1}(s) D(s) \int_{s-\tau(s)}^{s}(B(s, u)-D(u)) \varphi(u) d u d s \\
& +K(t) U(T) \int_{t}^{t+T} K^{-1}(s)[F(s) \varphi(s-\tau(s))+Q(s, \varphi(s), \varphi(s-\tau(s)))] d s
\end{aligned}
$$

and

$$
(\mathcal{S} \varphi)(t)=\int_{t-\tau(t)}^{t}(B(t, s)-D(s)) \varphi(s) d s .
$$

By a series of steps we will prove the fulfillment of $(i),(i i)$ and $(i i i)$ in Theorem 2.2. Since $\varphi \in \mathcal{C}_{T}$ and (2.2) hold, we have for $\varphi \in \mathcal{M}$

$$
(\mathcal{R} \varphi)(t+T)=(\mathcal{R} \varphi)(t) \text { and } \mathcal{R} \varphi \in C\left(\mathbb{R}, \mathbb{R}^{n}\right) \Longrightarrow(\mathcal{R} \mathcal{M}) \subset \mathcal{C}_{T}
$$

and

$$
(\mathcal{S} \varphi)(t+T)=(\mathcal{S} \varphi)(t) \text { and } \mathcal{S} \varphi \in C\left(\mathbb{R}, \mathbb{R}^{n}\right) \Longrightarrow(\mathcal{S M}) \subset \mathcal{C}_{T}
$$

Lemma 3.1. Suppose (2.2)-(2.5) hold. If $\mathcal{R}$ is defined by (3.3), then $\mathcal{R}$ is continuous and the image of $\mathcal{R}$ is contained in a compact set.

Proof. Let $\varphi_{n} \in \mathcal{M}$ where $n$ is a positive integer such that $\varphi_{n} \rightarrow \varphi$ as $n \rightarrow \infty$. Then

$$
\begin{gathered}
\left|\left(\mathcal{R} \varphi_{n}\right)(t)-(\mathcal{R} \varphi)(t)\right| \leq|K(t) U(T)| \int_{t}^{t+T}\left|K^{-1}(s)\right||D(s)| \\
\cdot \int_{s-\tau(s)}^{s}|B(s, u)-D(u)|\left|\varphi_{n}(u)-\varphi(u)\right| d u d s \\
+|K(t) U(T)| \int_{t}^{t+T}\left|K^{-1}(s)\right||F(s)|\left|\varphi_{n}(s-\tau(s))-\varphi(s-\tau(s))\right| d s \\
+|K(t) U(T)| \int_{t}^{t+T}\left|K^{-1}(s)\right| \mid Q\left(s, \varphi_{n}(s), \varphi_{n}(s-\tau(s))\right) \\
-Q(s, \varphi(s), \varphi(s-\tau(s))) \mid d s .
\end{gathered}
$$

Hence, the dominated convergence theorem implies,

$$
\lim _{n \rightarrow \infty}\left|\left(\mathcal{R} \varphi_{n}\right)(t)-(\mathcal{R} \varphi)(t)\right|=0 .
$$

So $\mathcal{R}$ is continuous. Next, we show that the image of $\mathcal{R}$ is contained in a compact set. So, let $\varphi_{n} \in \mathcal{M}$ where $n$ is a positive integer. Then (3.3) is 
equivalent to

$$
\begin{aligned}
\left(\mathcal{R} \varphi_{n}\right)(t) & =\int_{t}^{t+T}\left[K(s) U(T)^{-1} K(t)^{-1}\right]^{-1} D(s) \int_{s-\tau(s)}^{s}(B(s, u) \\
& -D(u)) \varphi_{n}(u) d u \\
& \int_{t}^{t+T}\left[K(s) U(T)^{-1} K(t)^{-1}\right]^{-1} F(s) \varphi_{n}(s-\tau(s)) d s \\
& \int_{t}^{t+T}\left[K(s) U(T)^{-1} K(t)^{-1}\right]^{-1} Q\left(s, \varphi_{n}(s), \varphi_{n}(s-\tau(s))\right) d s .
\end{aligned}
$$

By (2.5), we obtain

$$
\begin{aligned}
|Q(t, x, y)| & \leq|Q(t, x, y)-Q(t, 0,0)|+|Q(t, 0,0)| \\
& \leq k_{1} L+k_{2} L+\mu,
\end{aligned}
$$

where $\mu=\sup _{t \in[0, T]}|Q(t, 0,0)|$. Consequently

$$
\begin{aligned}
\left\|\left(\mathcal{R} \varphi_{n}\right)(\cdot)\right\| & \leq c \int_{0}^{T}\left[\alpha\|D\|\|B-D\| L+\|F\| L+k_{1} L+k_{2} L+\mu\right] d s \\
& =c T\left[\alpha\|D\|\|B-D\| L+\|F\| L+k_{1} L+k_{2} L+\mu\right]=E,
\end{aligned}
$$

where

$$
\begin{aligned}
\alpha & =\sup _{t \in[0, T]}|\tau(t)|, \\
c & =\sup _{t \in[0, T]}\left(\sup _{s \in[t, t+T]}\left|\left[K(s) U(T)^{-1} K(t)^{-1}\right]^{-1}\right|\right) .
\end{aligned}
$$

Second, we calculate $\left(\mathcal{R} \varphi_{n}\right)^{\prime}(t)$ and show that it is uniformly bounded. By making use of (2.2) we obtain, by taking the derivative in (3.3) that

$$
\begin{gathered}
\left(\mathcal{R} \varphi_{n}\right)^{\prime}(t)=K^{\prime}(t) U(T) \int_{t}^{t+T} K^{-1}(s)\left[F(s) \varphi_{n}(s-\tau(s))\right. \\
\left.+Q\left(s, \varphi_{n}(s), \varphi_{n}(s-\tau(s))\right)\right] d s \\
+K^{\prime}(t) U(T) \int_{t}^{t+T} K^{-1}(s) D(s) \int_{s-\tau(s)}^{s}(B(s, u)-D(u)) \varphi_{n}(u) d u d s \\
+K(t) U(T)\left[K^{-1}(t+T)-K^{-1}(t)\right]\left[F(t) \varphi_{n}(t-\tau(t))\right. \\
\left.+Q\left(t, \varphi_{n}(t), \varphi_{n}(t-\tau(t))\right)\right] \\
+K(t) U(T)\left[K^{-1}(t+T)-K^{-1}(t)\right] D(t) \int_{t-\tau(t)}^{t}(B(t, s)-D(s)) \varphi_{n}(s) d s .
\end{gathered}
$$


Since

$$
K^{\prime}(t)=D(t) K(t)
$$

and noting that $K^{-1}(t+T)=e^{-B T} K^{-1}(t)$, we have

$$
K^{-1}(t+T)-K^{-1}(t)=e^{-B T} K^{-1}(t)-K^{-1}(t)=\left(K^{-1}(T)-1\right) K^{-1}(t) .
$$

A substitution of (3.8) and (3.9) into (3.7) yields

$$
\begin{aligned}
\left(\mathcal{R} \varphi_{n}\right)^{\prime}(t) & =D(t)\left(\mathcal{R} \varphi_{n}\right)(t)+D(t) \int_{t-\tau(t)}^{t}(B(t, s)-D(s)) \varphi_{n}(s) d s \\
& +F(t) \varphi_{n}(t-\tau(t))+Q\left(t, \varphi_{n}(t), \varphi_{n}(t-\tau(t))\right) .
\end{aligned}
$$

Hence

$$
\left\|\left(\mathcal{R} \varphi_{n}\right)^{\prime}(\cdot)\right\| \leq\|D\| E+\frac{E}{c T} .
$$

Thus, the sequence $\left(\mathcal{R} \varphi_{n}\right)$ is uniformly bounded and equicontinuous. Therefore, by Ascoli-Arzela's theorem $\mathcal{R}(\mathcal{M})$ is relatively compact.

Lemma 3.2. Suppose (2.2) and (2.3) hold and

$$
\alpha\|B-D\|<1 \text {. }
$$

If $\mathcal{S}$ is defined by (3.4), then $\mathcal{S}$ is a contraction.

Proof. Let $\mathcal{S}$ be defined by (3.4). Then for $\varphi_{1}, \varphi_{2} \in \mathcal{M}$ we have

$$
\begin{aligned}
& \left|\left(\mathcal{S} \varphi_{1}\right)(t)-\left(\mathcal{S} \varphi_{2}\right)(t)\right| \\
& =\left|\int_{t-\tau(t)}^{t}(B(t, s)-D(s)) \varphi_{1}(s) d s-\int_{t-\tau(t)}^{t}(B(t, s)-D(s)) \varphi_{2}(s) d s\right| \\
& \leq \alpha\|B-D\|\left\|\varphi_{1}-\varphi_{2}\right\| .
\end{aligned}
$$

Hence $\mathcal{S}$ is contraction by (3.10).

Theorem 3.1. Suppose the assumptions of Lemmas 3.1 and 3.2 hold. Let $L$ be a positive constant satisfying the inequality

$$
c T\left[\alpha\|D\|\|B-D\| L+\|F\| L+k_{1} L+k_{2} L+\mu\right]+\alpha\|B-D\| L \leq L .
$$

Then (1.2) has a T-periodic solution.

Proof. By Lemma 3.1, $\mathcal{R}: \mathcal{M} \rightarrow \mathcal{C}_{T}$ is continuous and $\mathcal{R}(\mathcal{M})$ is contained in a compact set. Also, from Lemma 3.2, the mapping $\mathcal{S}: \mathcal{M} \rightarrow \mathcal{C}_{T}$ is a contraction. Next, we show that if $\varphi, \phi \in \mathcal{M}$, we have $\|\mathcal{R} \varphi+\mathcal{S} \phi\| \leq L$. Let $\varphi, \phi \in \mathcal{M}$ with $\|\varphi\|,\|\phi\| \leq L$. Then

$$
\begin{aligned}
& \|(\mathcal{R} \varphi)(\cdot)+(\mathcal{S} \phi)(\cdot)\| \\
& \leq c T\left[\alpha\|D\|\|B-D\| L+\|F\| L+k_{1} L+k_{2} L+\mu\right]+\alpha\|B-D\| L \\
& \leq L .
\end{aligned}
$$


Clearly, all the hypotheses of the Krasnoselskii's theorem are satisfied. Thus there exists a fixed point $z \in \mathcal{M}$ such that $z=\mathcal{R} z+\mathcal{S} z$. By Lemma 2.2 this fixed point is a solution of (1.2). Hence (1.2) has a $T$-periodic solution.

Theorem 3.2. Suppose the assumptions (2.2)-(2.5) hold. If

$$
c T\left[\alpha\|D\|\|B-D\|+|F|+k_{1}+k_{2}\right]+\alpha\|B-D\|<1,
$$

then equation (1.2) has a unique T-periodic solution.

Proof. Let the mapping $\mathcal{H}$ be given by (3.2). For $\varphi_{1}, \varphi_{2} \in \mathcal{C}_{T}$, we have

$$
\begin{gathered}
\left|\left(\mathcal{H} \varphi_{1}\right)(t)-\left(\mathcal{H} \varphi_{2}\right)(t)\right| \leq\left|\int_{t-\tau(t)}^{t}(B(t, s)-D(s))\left(\varphi_{1}(s)-\varphi_{2}(s)\right) d s\right| \\
+\int_{t}^{t+T}\left|\left[K(s) U(T)^{-1} K(t)^{-1}\right]^{-1}\right| \\
\cdot|D(s)| \int_{s-\tau(s)}^{s}|B(s, u)-D(u)|\left|\varphi_{1}(u)-\varphi_{2}(u)\right| d s \\
+\int_{t}^{t+T}\left|\left[K(s) U(T)^{-1} K(t)^{-1}\right]^{-1}\right||F(s)|\left|\varphi_{1}(s-\tau(s))-\varphi_{2}(s-\tau(s))\right| d s \\
\quad+\int_{t}^{t+T}\left|\left[K(s) U(T)^{-1} K(t)^{-1}\right]^{-1}\right| \\
\cdot k_{1}\left|\varphi_{1}(s)-\varphi_{2}(s)\right|+k_{2}\left|\varphi_{1}(s-\tau(s))-\varphi_{2}(s-\tau(s))\right| d s \\
\leq\left\{c T\left[\alpha\|D\|\|B-D\|+\|F\|+k_{1}+k_{2}\right]+\alpha\|B-D\|\right\}\left\|\varphi_{1}-\varphi_{2}\right\| .
\end{gathered}
$$

Since (3.11) hold, the contraction mapping principle completes the proof.

Corollary 3.1. Suppose (2.2), (2.3), (2.4) and (3.10) hold. Let $\mathcal{M}$ defined by (3.1). Suppose there are positive constants $k_{1}^{*}, k_{2}^{*}$, such that for $x, y, z$ and $w \in \mathcal{M}$, we have

$$
|Q(t, x, y)-Q(t, z, w)| \leq k_{1}^{*}\|x-z\|+k_{2}^{*}\|y-w\|
$$

and

$$
c T\left[\alpha\|D\|\|B-D\| L+\|F\| L+k_{1}^{*} L+k_{2}^{*} L+\mu\right]+\alpha\|B-D\| L \leq L .
$$

Then (1.2) has a T-periodic solution in $\mathcal{M}$. Moreover, if

$$
c T\left[\alpha\|D\|\|B-D\|+\|F\|+k_{1}^{*}+k_{1}^{*}\right]+\alpha\|B-D\|<1,
$$

then (1.2) has a unique solution in $\mathcal{M}$.

Proof. Let the mapping $\mathcal{H}$ defined by (3.2). Then the proof follow immediately from Theorem 3.1 and Theorem 3.2. 


\section{Application in two-Dimensional model}

Consider the following system

$$
\begin{aligned}
\left(\begin{array}{l}
x_{1}(t) \\
x_{2}(t)
\end{array}\right)^{\prime} & =\int_{t-\lambda_{1}}^{t}\left(\begin{array}{cc}
\cos (t-s) & -\sin (t-s) \\
\sin (t-s) & \cos (t-s)
\end{array}\right)\left(\begin{array}{c}
x_{1}(s) \\
x_{2}(s)
\end{array}\right) d s \\
& +\left(\begin{array}{cc}
\lambda_{2} \cos (t) x_{1}(t)-\lambda_{3} x_{1}\left(t-\lambda_{1}\right)+\lambda_{4} \sin t
\end{array}\right),
\end{aligned}
$$

where $\tau(t)=\lambda_{1}$ be a positive constant and

$$
\begin{aligned}
A(t, s) & =\left(\begin{array}{cc}
\cos (t-s) & -\sin (t-s) \\
\sin (t-s) & \cos (t-s)
\end{array}\right), \\
Q(t, x, y) & =\left(\begin{array}{c}
0 \\
\lambda_{2} \cos (t) x_{1}-\lambda_{3} y_{1}+\lambda_{4} \sin t
\end{array}\right),
\end{aligned}
$$

with $x=\left(x_{1}, x_{2}\right)^{t}, y=\left(y_{1}, y_{2}\right)^{t}$.

Consider the Banach space $\left(\mathcal{C}_{2 \pi},\|\cdot\|\right)$,

$$
\mathcal{C}_{2 \pi}=\left\{\phi: \phi \in C\left(\mathbb{R}, \mathbb{R}^{2}\right), \phi(t+2 \pi)=\phi(t), t \in \mathbb{R}\right\}
$$

and the closed bounded convex subset of $\mathcal{C}_{2 \pi}$,

$$
\mathcal{M}=\left\{\varphi \in \mathcal{C}_{2 \pi},\|\varphi\| \leq L\right\} .
$$

Let $\varphi=\left(\varphi_{1}, \varphi_{2}\right)^{t}, \phi=\left(\phi_{1}, \phi_{2}\right)^{t}$. Then for $\varphi, \phi \in \mathcal{M}$ we have

$$
\begin{aligned}
& \|Q(\cdot, \varphi(\cdot), \varphi(\cdot-\tau(\cdot)))-Q(\cdot, \phi(\cdot), \phi(\cdot-\tau(\cdot)))\| \\
& \leq \lambda_{2}\|\varphi-\phi\|+\lambda_{3}\|\varphi-\phi\| .
\end{aligned}
$$

Hence $k_{1}^{*}=\lambda_{2}, k_{2}^{*}=\lambda_{3}, \alpha=\lambda_{1}, \mu=\lambda_{4}$ and

$$
\begin{aligned}
B\left(t, t-\lambda_{1}\right) & =\int_{t}^{t-\lambda_{1}} A\left(u, t-\lambda_{1}\right) d u \\
& =\int_{t}^{t-\lambda_{1}}\left(\begin{array}{cc}
\cos \left(u-t+\lambda_{1}\right) & -\sin \left(u-t+\lambda_{1}\right) \\
\sin \left(u-t+\lambda_{1}\right) & \cos \left(u-t+\lambda_{1}\right)
\end{array}\right) d u \\
& \left.=\left[\begin{array}{cc}
\sin \left(u-t+\lambda_{1}\right) & \cos \left(u-t+\lambda_{1}\right) \\
-\cos \left(u-t+\lambda_{1}\right) & \sin \left(u-t+\lambda_{1}\right)
\end{array}\right)\right]_{t}^{t-\lambda_{1}} \\
& =\left(\begin{array}{cc}
-\sin \lambda_{1} & 1-\cos \lambda_{1} \\
-1+\cos \lambda_{1} & -\sin \lambda_{1}
\end{array}\right) .
\end{aligned}
$$

Then

$$
D(t)=B\left(t, t-\lambda_{1}\right)
$$

and

$$
F(t)=D(t)-D\left(t-\lambda_{1}\right)=\left(\begin{array}{ll}
0 & 0 \\
0 & 0
\end{array}\right) .
$$


Since the matrix $D(\cdot)$ has eigenvalues with non-zero real parts, the system $x^{\prime}(t)=D(t) x(t)$ is noncritical where $D(\cdot)$ is given as above.

By the above calculation we obtain

$$
c 2 \pi\left[\lambda_{1}\|D\|\|B-D\| L+\lambda_{2} L+\lambda_{3} L+\lambda_{4}\right]+\lambda_{1}\|B-D\| L \leq L
$$

for $\lambda_{i}, 1 \leq i \leq 4$ small enough. Then (4.1) has a $2 \pi$-periodic solution by Corollary 3.1. Moreover,

$$
c 2 \pi\left[\lambda_{1}\|D\|\|B-D\|+\lambda_{2}+\lambda_{3}\right]+\lambda_{1}\|B-D\|<1
$$

is satisfied for $\lambda_{i}, 1 \leq i \leq 4$ small enough. Then (4.1) has a unique $2 \pi$ periodic solution by Corollary 3.1 .

Acknowledgments. The authors would like to express their sincere thanks to the anonymous referee for his/her careful reading of our manuscript and the helpful report.

\section{REFERENCES}

[1] T. A. Burton, A fixed point theorem of Krasnoselskii, Appl. Math. Lett., 11 (1) (1998), $85-88$.

[2] T. A. Burton, Stability by Fixed Point Theory for Functional Differential Equations, Dover Publications, New York, 2006.

[3] T. R. Ding, R. Iannacci and F. Zanolin, On periodic solutions of sublinear Duffing equations, J. Math. Anal. Appl., 158 (1991) 316-332.

[4] J. Hale, Ordinary Differential Equations, Robert E. Krieger Publishing Company, New York, 1980.

[5] J. K. Hale and S. M. Verduyn Lunel, Introduction to Functional Differential Equations, Springer Verlag, New York,1993.

[6] K. Gopalsamy, X. He, and L. Wen, On a periodic neutral logistic equation, Glasgow Math., J. 33 (1991), 281-286.

[7] K. Gopalsamy and B. G. Zhang, On a neutral delay-logistic equation, Dynam. Stability Systems, 2 (1988), 183-195.

[8] I. Gyori and F. Hartung, Preservation of stability in a linear neutral differential equation under delay perturbations, Dyn. Syst. Appl., 10 (2001), 225-242.

[9] I. Gyori and G. Ladas, Positive solutions of integro-differential equations with unbounded delay, J. Integral Equations Appl., 4 (1992), 377-390.

[10] M. N. Islam and Y. N. Raffoul, Periodic solutions of neutral nonlinear system of differential equations with functional delay, J. Math. Anal. Appl., 331 (2007) 11751186.

[11] C. H. Jin and J. W. Luo, Stability of an integro-differential equation, Comput. Math. Appl., 57 (2009) 1080-1088.

[12] C. H. Jin and J. W. Luo, Stability in functional differential equations established using fixed point theory, Nonlinear Anal., 68 (2008) 3307-3315.

[13] C. H. Jin and J. W. Luo, Fixed points and stability in neutral differential equations with variable delays, Proc. Amer. Math. Soc., 136 (3) (2008), 909-918.

[14] L. Y. Kun, Periodic solutions of a periodic neutral delay equation, J. Math. Anal. Appl., 214 (1997), 11-21. 
[15] V. Lakshmikantham and S. G. Deo, Methods of Variation of Parameters for Dynamical Systems, Gordon and Breach Science Publishers, Australia, 1998.

[16] M. Maroun and Y. N. Raffoul, Periodic solutions in nonlinear neutral difference equations with functional delay, J. Korean Math. Soc., 42 (2005), 255-268.

[17] D. R. Smart, Fixed Point Theorems, Cambridge University Press, 1980. 2.4

[18] R. Weikard, Floquet theory for linear differential equations with meromorphic solutions, Electron. J. Qual. Theory Differ. Equ., 8 (2000), 1-6.

[19] E. Yankson, Periodicity in a system of differential equations with finite delay, Casp. J. Math. Sci., In press.

(Received: February 25, 2014)

(Revised: May 26, 2014)
M. B. Mesmouli, A. Ardjouni and A. Djoudi Applied Mathematics Laboratory Faculty of Sciences

Department of Mathematics

University Annaba

P.O. Box 12

Annaba 23000, Algeria

mesmoulimouataz@hotmail.com

abd_ardjouni@yahoo.fr

adjoudi@yahoo.com

Abdelouaheb Ardjouni - 2. address:

Faculty of Sciences and Technology

Department of Mathematics and Informatics University Souk Ahras

P.O. Box 1553

Souk Ahras, 41000, Algeria 